\title{
IRS2 mutations linked to invasion in pleomorphic invasive lobular carcinoma
}

\author{
Sha Zhu, ${ }^{1}$ B. Marie Ward, ${ }^{2}$ Jun Yu, ${ }^{1}$ Asia N. Matthew-Onabanjo, ${ }^{1}$ Jenny Janusis, ${ }^{1}$ Chung-Cheng Hsieh, \\ Keith Tomaszewicz, ${ }^{3}$ Lloyd Hutchinson, ${ }^{3}$ Lihua Julie Zhu, ${ }^{1,4,5}$ Dina Kandil, ${ }^{3}$ and Leslie M. Shaw ${ }^{1}$ \\ ${ }^{1}$ Department of Molecular, Cell and Cancer Biology, ${ }^{2}$ Department of Surgery, ${ }^{3}$ Department of Pathology, ${ }^{4}$ Department of \\ Molecular Medicine, and ${ }^{5}$ Program in Bioinformatics and Integrative Biology, University of Massachusetts Medical School, \\ Worcester, Massachusetts, USA.
}

Pleomorphic invasive lobular carcinoma (PILC) is an aggressive variant of invasive lobular breast cancer that is associated with poor clinical outcomes. Limited molecular data are available to explain the mechanistic basis for PILC behavior. To address this issue, targeted sequencing was performed to identify molecular alterations that define PILC. This sequencing analysis identified genes that distinguish PILC from classic ILC and invasive ductal carcinoma by the incidence of their genomic changes. In particular, insulin receptor substrate 2 (IRS2) is recurrently mutated in PILC, and pathway analysis reveals a role for the insulin receptor (IR)/insulin-like growth factor-1 receptor (IGF1R)/IRS2 signaling pathway in PILC. IRS2 mutations identified in PILC enhance invasion, revealing a role for this signaling adaptor in the aggressive nature of PILC.

Authorship note: SZ and BMW contributed equally to this work.

Conflict of interest: The authors have declared that no conflict of interest exists.

Submitted: September 12, 2017 Accepted: March 14, 2018 Published: April १९, 2018

\section{Reference information:} JCI Insight. 2018;3(8):e97398. https://doi.org/10.1172/jci. insight. 97398.

\section{Introduction}

Invasive breast cancer is a heterogenous disease, with the majority of tumors diagnosed as either invasive ductal carcinoma (IDC; 80\%) or invasive lobular carcinoma (ILC; 8-14\%). Pleomorphic ILC (PILC) is a relatively recent, formally recognized variant of invasive lobular breast cancer that represents approximately $10 \%$ of lobular tumors (1). PILC shares histologic and molecular hallmarks with classic ILC (CILC), particularly its distinctive infiltrative growth pattern, lack of E-cadherin expression, and common chromosomal alterations (1-3). However, PILC differs from CILC in its greater degree of cellular atypia and nuclear pleomorphism, which is more similar to high-grade IDC. Molecular prognostic features of PILC also distinguish this lesion from CILC. Specifically, PILC tends to be ER/PR negative and HER2 positive more frequently than $\operatorname{CILC}(3,4)$.

PILC typically presents at advanced stages and is associated with larger tumor size, greater presence of lymphovascular invasion, more frequent regional axillary lymph node involvement, and a higher rate of distant metastasis when compared with CILC (1). These poor prognostic factors translate into reduced clinical outcomes with short relapse times, a higher risk of recurrence, and decreased overall survival (5). A tendency toward lower complete response rates of ILC to adjuvant chemotherapy has been observed when compared with IDC (4). However, given the relatively recent clinical recognition of PILC, response data from independent studies on PILC are lacking. A greater understanding of PILC biology is needed to explain its more aggressive behavior and to determine optimal clinical management.

The aim of this study was to establish a molecular profile of pleomorphic lobular carcinoma that could inform the mechanistic basis of this breast cancer variant. Our data identify gain-of-function IRS2 mutations that reveal a role for the insulin/IGF-1 signaling pathway in PILC and its potential to facilitate the invasive nature of these tumors.

\section{Results}

Identification of recurrently mutated genes in PILC. PILC tumors (Figure 1, A and B) and their paired normal tissues were subjected to targeted exome sequencing across the protein-coding exons and flanking splice sites of the Beijing Genomics Institute TumorCare gene panel. The clinicopathological features of the data set are presented in Table 1. The TumorCare gene panel includes 1,053 genes that were selected based upon previous reports of being clinically actionable cancer genes, harboring high frequency alterations in the Catalog of Somatic Mutations in Cancer (COSMIC) database (6), or playing a key role in pathways 
Table 1. Clinical characteristics of study cases

\begin{tabular}{|c|c|}
\hline Characteristics & No. $(\%, n=16)^{\mathrm{A}}$ \\
\hline \multicolumn{2}{|l|}{ Age at diagnosis, yr } \\
\hline Mean \pm SD & $67.5 \pm 13.1$ \\
\hline$\geq 60$ & $13(81.3)$ \\
\hline$<60$ & $3(18.7)$ \\
\hline \multicolumn{2}{|l|}{ Tumor size, $\mathrm{cm}$} \\
\hline Mean \pm SD & $2.5 \pm 1.5$ \\
\hline$\geq 2$ & $8(50)$ \\
\hline$<2$ & $8(50)$ \\
\hline \multicolumn{2}{|l|}{ Tumor stage } \\
\hline 0 & $0(0)$ \\
\hline 1 & $5(31.3)$ \\
\hline II & $10(62.5)$ \\
\hline III & $1(6.2)$ \\
\hline \multicolumn{2}{|l|}{ Lymph node status } \\
\hline Positive & $6(37.5)$ \\
\hline Negative & $10(62.5)$ \\
\hline \multicolumn{2}{|l|}{ Hormone receptor status } \\
\hline${ }^{\mathrm{B}}$ ER positive & $13(81.3)$ \\
\hline 'PR positive & $10(62.5)$ \\
\hline \multicolumn{2}{|l|}{ HER2 status } \\
\hline Positive & $4(25)$ \\
\hline Negative & $11(68.8)$ \\
\hline DEquivocal & $1(6.2)$ \\
\hline \multicolumn{2}{|l|}{ Treatment } \\
\hline Lumpectomy & $4(25)$ \\
\hline Mastectomy & $11(68.8)$ \\
\hline Adjuvant radiation & $8(50)$ \\
\hline Adjuvant chemotherapy & $7(43.7)$ \\
\hline $\begin{array}{c}\text { Adjuvant endocrine } \\
\text { therapy }\end{array}$ & $10(62.5)$ \\
\hline Anastrozole & $7(43.7)$ \\
\hline Tamoxifen & $3(18.7)$ \\
\hline
\end{tabular}

${ }^{\text {A}}$ Values are shown, with percentages provided parenthetically, except when values are shown as mean $\pm \mathrm{SD}$. ${ }^{\mathrm{B}} \mathrm{ER}$, estrogen receptor; ${ }^{\mathrm{C} P R}$, progesterone receptor; HER2, human epidermal growth factor receptor 2. ${ }^{\mathrm{B}} \mathrm{By}$ IHC and FISH. associated with cancer (Supplemental Table 1; supplemental material available online with this article; https://doi.org/10.1172/jci.insight.97398DS1). Total somatic mutation events and copy number variations (CNVs) for each sample are shown in Figure $1, \mathrm{D}$ and $\mathrm{E}$, respectively. There was no strong positive correlation between the total number of molecular alterations in each sample and the depth of coverage (Figure 1F). The somatic mutations and CNVs that occurred in PILC are provided in Supplemental Tables 2 and 3, respectively.

To identify genes that are functionally important for PILC, a filtered data set of molecular alterations that met specific criteria was established that included (a) all nonsense, indel, and splice-site mutations; (b) copy number gains and losses; (c) missense mutations present in the COSMIC v77 database; and (d) novel missense mutations not present in COSMIC that were predicted to have protein-altering functional consequences by comprehensive computational prediction tools (Supplemental Table 4). Based upon these criteria, a total of 798 functional somatic mutation events were identified in 426 genes, with an average of 46.9 protein-altering mutations (range of 9-137) per sample (Figure 1G). Genes that were recurrently mutated in PILC ( $\geq 4$ samples) are shown in Figure $1 \mathrm{H}$.

IDC and ILC have distinct histological characteristics and clinical outcomes, and molecular alterations that distinguish these breast cancer variants have been identified (7-9). Comparison of our data with published comprehensive genomic studies of ILC and IDC revealed that PILC and ILC share many of the significant alterations that are associated with ILC and confirmed molecular differences that distinguish ILC from IDC (7-9) (Figure 2, A and B, and Supplemental Table 5). PIK3CA, the catalytic subunit of PI3K (10), was mutated at a high frequency (Figure $1 \mathrm{H})$, with known gainof-function missense mutations in 9 of 17 (53\%) of the PILC samples (Figure 2C). Loss of E-cadherin expression is a defining hallmark of lobular neoplasias (2), and negative E-cadherin staining was confirmed for all PILC samples (Figure 1C). CDH1, which encodes for E-cadherin, was altered in 10 of 17 (59\%) of the PILC samples (Figure $1 \mathrm{H}$ ), a mutation frequency similar to that reported previously for ILC (Figure 2B and Supplemental Table 5) (7-9). All of the CDH1 mutations were either frameshift indels or nonsense mutations that would be predicted to result in loss of protein expression (Figure 2C). Additional genes with similar mutation frequencies in PILC and ILC, and that distinguish ILC from IDC, include TP53, RUNX1, GATA3, TBX3, and $M Y C$ (Figure 2, A and B)

Molecular alterations that distinguish PILC from ILC were identified in our study (Figure 2B). Amplification or mutation of both HER2 (also known as ERBB2) and HER3 (also known as ERBB3) occurred more frequently in PILC than in ILC (Figure 2B and Supplemental Table 5). Several additional genes were mutated at a markedly higher frequency in our PILC cohort when compared with ILC, and they represent PILC-associated molecular alterations. These genes include KMT2C, MAP3K1, IRS2, NCOR1, NF1, and TBX3 (Figure 2B). The incidence of PTEN molecular alterations may also distinguish PILC from ILC. Although the frequency of PTEN mutations reported for ILC varies among published studies (1.4\%-13\%) (Supplemental Table 5), molecular alterations were not identified in either our study or a previous whole-exome sequencing (WES) analysis of 8 PILC tumors (11). In support of a selective association of PTEN loss with CILC, mice with combined loss of Cdh1 and Pten develop mammary tumors that model human CILC (12). Of the recurrently mutated genes identified in our study, only TP53, CDH1, and PI3KCA were reported in the previous PILC WES (Supplemental Table 5) (11). The mutation detection differences in our studies likely reflect both the greater depth of coverage provided by our targeted sequencing and the higher tumor content of our samples ( $>60 \%$ vs. $30 \%$ ).

The IRS2 signaling pathway is associated with PILC. To identify genes and pathways that drive the more aggressive nature of PILC tumors, we analyzed our somatic mutation data using MUtations For Functional Impact on Network Neighbors (MUFFINN) (13). This program integrates mutational data for individual genes and their network neighbors to predict functional impact. Of relevance for our study, MUFFINN shows high sensitivity for small sample sizes. We analyzed our data using the direct-neighbor max approach 

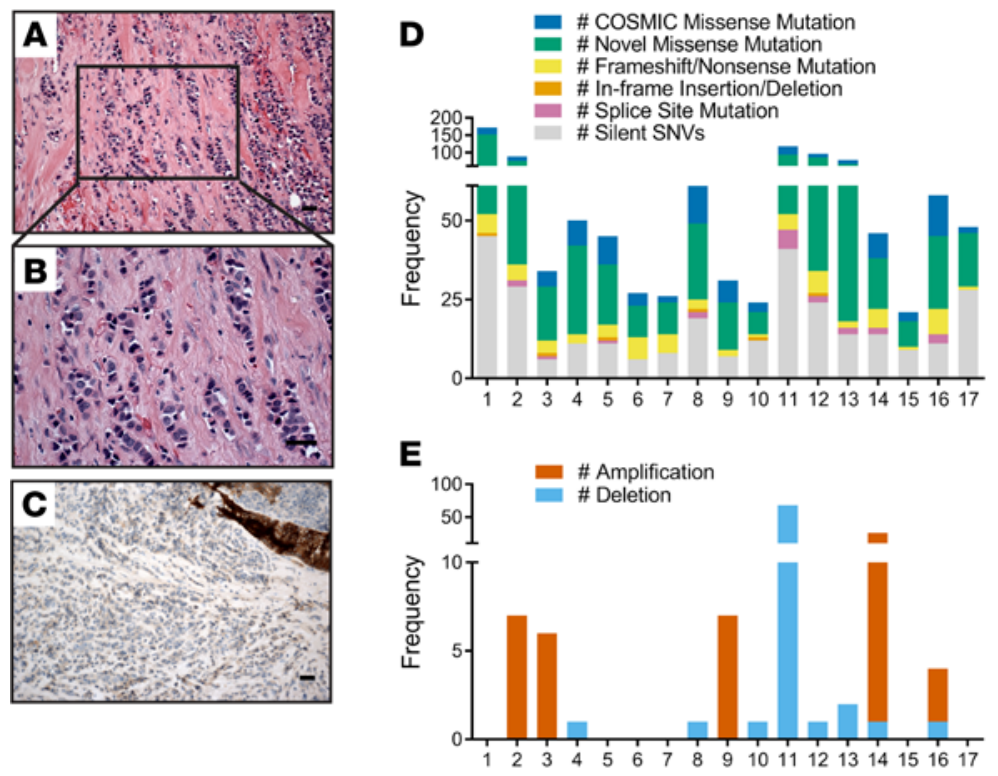

$\mathbf{F}$

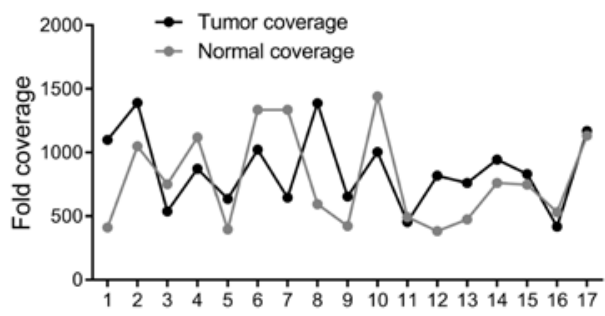

G

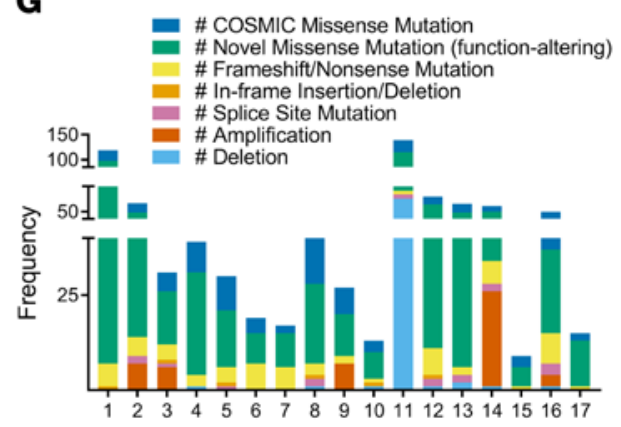

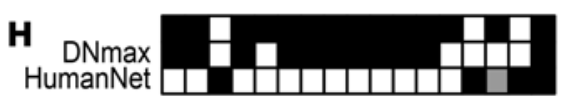

ER Status PR Status Her2 Status

0.989 0.993 믈 PIK3CA $(53 \%)$

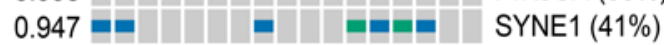
0.738 KMT2C (35\%) $0.738+\square=$ MAP3K1 $(35 \%)$ 0.717 믐 $0.982=-1=0$ $0.721=\quad[\quad=\quad$ KAT6B $(29 \%)$

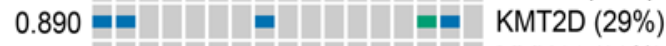
0.856 E 0.658 들 0.804 -

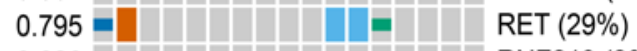
0.626 E 0.874 -

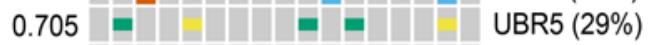
0.764 CREBBP (24\%) $0.586=\square-\square=$ DST $(24 \%)$ $0.442=\square-\square=\operatorname{EPPK} 1(24 \%)$ 0.746 - ERBB3 $(24 \%)$ $0.706=\square-\square=2$ $0.764+2-\quad$ KMT2A $(24 \%)$

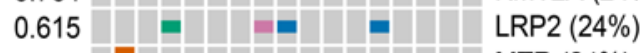
0.587 - $\square+\square=$ MTR $(24 \%)$ $0.653+\square \quad+\quad$ NCOR1 $(24 \%)$

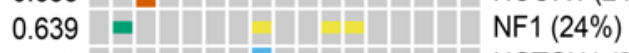
0.640 E $\quad+\quad+\quad$ NOTCH1 $(24 \%)$

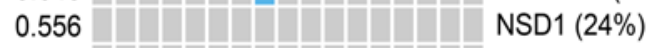
$0.660=\quad+\quad=\quad$ PARP1 $(24 \%)$ $0.618=-1=\operatorname{PBRM} 1(24 \%)$ $0.588=\square=\operatorname{PTPRD}(24 \%)$

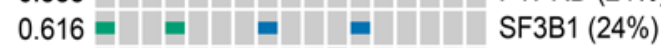
$0.735+\square=$ SMARCA4 $(24 \%)$ $0.503+\square=\operatorname{TBX} 3(24 \%)$ 0.526 USP9X $(24 \%)$

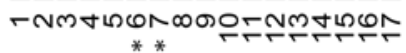

$\begin{array}{ll}\text { Mutation Status } & \text { ER/PR/Her2 Status } \\ \begin{array}{ll}\text { cosmiC Missense Mutation } \\ \text { Novel Missense Mutation }\end{array} \\ \begin{array}{l}\text { Frameshift/Nonsense } \\ \text { In-frame Deletion }\end{array} & \text { Positive } \\ \text { Negative } \\ \text { Equice Site Mutation } \\ \text { Amplification } \\ \text { Deletion }\end{array}$

Figure 1. Molecular profile of pleomorphic invasive lobular carcinoma. (A and B) Representative images of H\&E-stained pleomorphic invasive lobular carcinoma (PILC). (C) Representative image of E-cadherin staining in PILC. (D) Total mutation events across the PILC tumors ( $n=17)$. (E) Distribution of copy number variant types ( $\log _{2}$ ratio $\geq 1$ or $\left.\leq-1\right)$ across the PILC tumors. (F) Mean coverage of sequenced PILC tumors and their matched normal controls. (G) Filtered functional somatic alteration events identified in the PILC tumors. (H) Oncoprint heatmaps of recurrently altered genes in PILC. MUtations For Functional Impact on Network Neighbors (MUFFINN) prediction scores are shown on left. Asterisk indicate samples from the same patient. Scale bar: $20 \mu \mathrm{m}$.

with the HumanNet gene network (13). Prediction scores generated by this analysis range from 0 to 1 , with a larger value indicating higher significance. Scores are indicated in the left column of the Oncoprint plot in Figure $1 \mathrm{H}$, and the top 10 genes predicted to contribute to PILC are shown in Table 2. PIK3CA and CDH1, genes known to play an important role in breast cancer and ILC, respectively, were assigned the highest predictive scores. IRS2 was ranked third, with genes encoding its upstream receptors insulin-like growth factor-1 receptor $(I G F 1 R)$ and IR (encoded by INSR), also ranked within the top 10 genes (Figure 2C). Network analysis for IRS2 revealed additional molecular alterations in both upstream regulators and downstream effectors of IRS2 that support a role for this signaling adaptor in PILC (Figure 2D). 
A

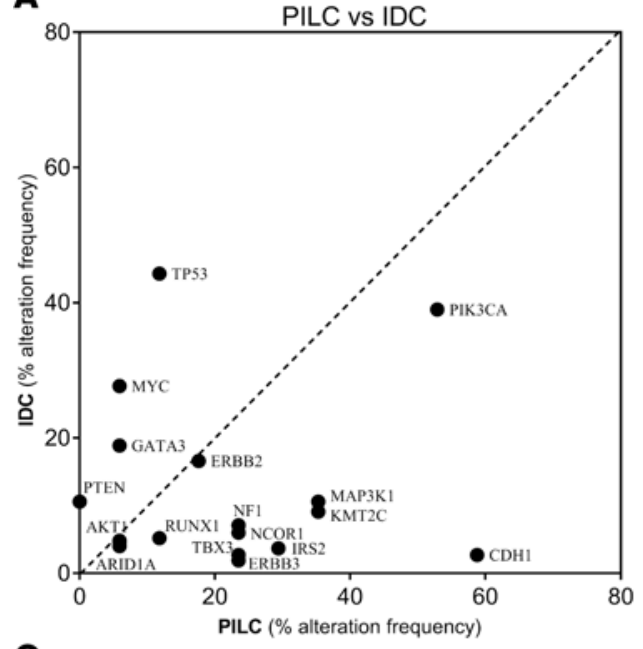

C

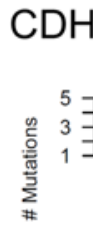
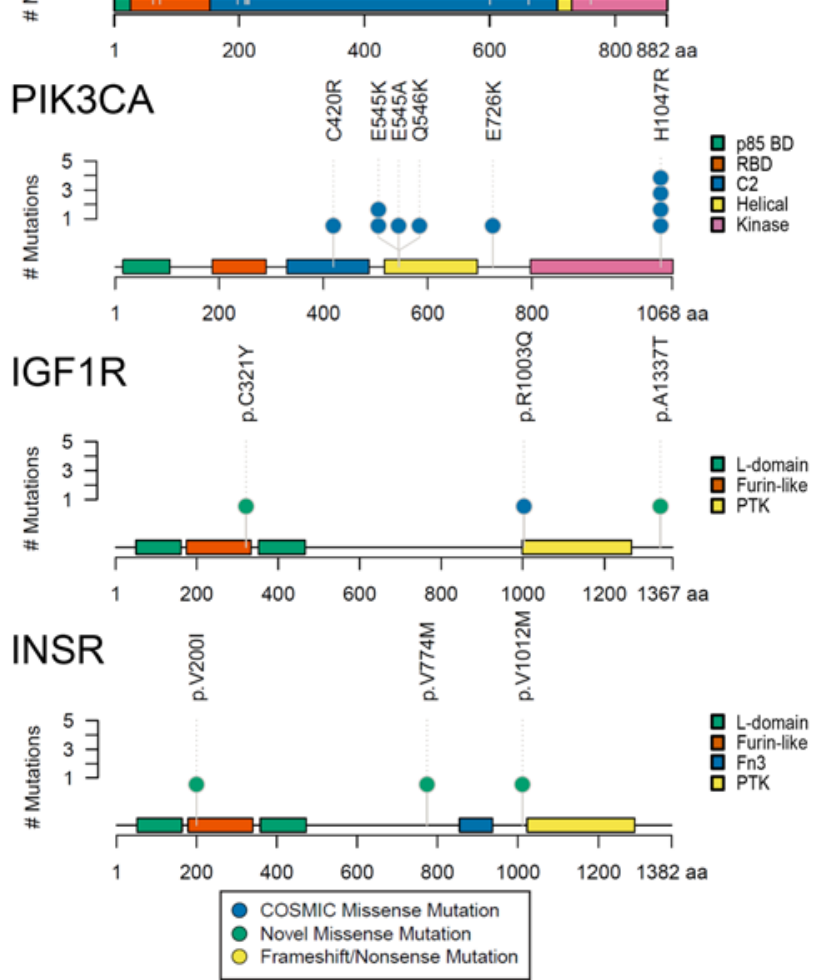

B
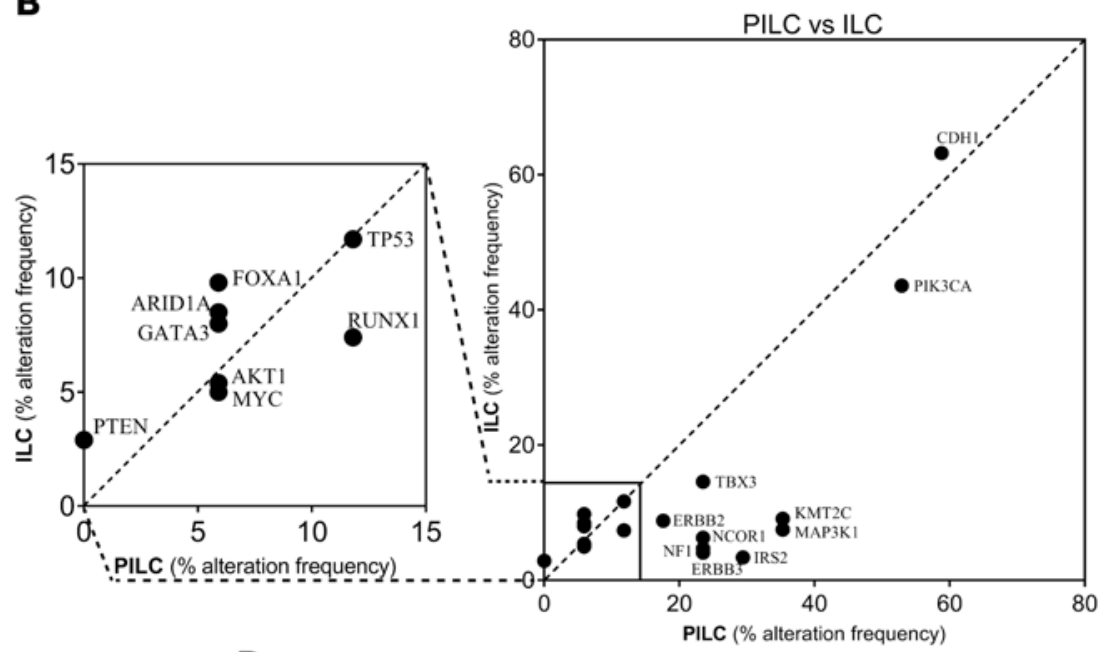

D

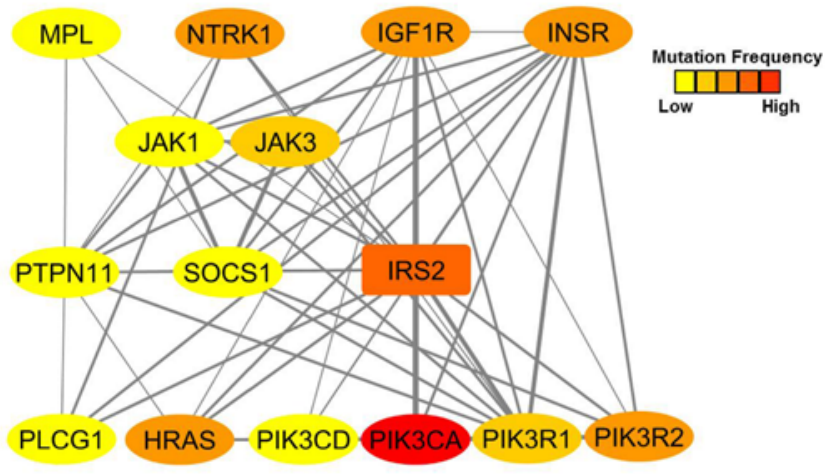

E

IRS-related events triggered by IGF1R PI3K/AKT Signaling in Cancer Signaling by SCF-KIT GAB1 signalosome NGF signalling via TRKA from the plasma membrane Signaling by VEGF

Insulin receptor signalling cascade PI3K events in ERBB4 signaling PI3K events in ERBB2 signaling Downstream signaling of activated FGFR Signaling by EGFR Interleukin-6 signaling EPHA-mediated growth cone collapseSignalling to RAS HSF1-dependent transactivation SUMOylation of DNA damage response and repair proteins Chromatin modifying enzymes
Transcriptional Regulation by TP53 NOTCH1 Intracellular Domain Regulates Transcription-

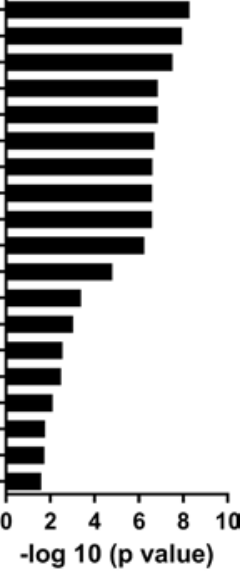

Figure 2. Analysis of pleomorphic invasive lobular carcinoma-specific molecular alterations. Comparison of molecular alterations identified in (A) pleomorphic invasive lobular carcinoma (PILC) $(n=17)$ versus invasive ductal carcinoma (IDC) ( $n=481)$ or (B) PILC ( $n=17)$ versus ILC ( $n=684)$. (C) Lollipop plots depicting PILC somatic mutations in PIK3CA, CDH1, IGF1R, and INSR (R Package trackviewer, http://bioconductor.org/packages/release/ bioc/html/trackViewer.html). Protein-coding sequences and conserved domains derived from uniProt (25). (D) Network connection map of IRS2 and its direct neighbors, as predicted by MUtations For Functional Impact on Network Neighbors (MUFFINN). Gene-gene interactions are visualized by the CytoScape program (51). (E) Reactome pathway enrichment terms according to $-\log _{10}(P$ value) for PILC. 
Table 2. Top ranked genes by MUFFINN analysis

\begin{tabular}{cccc}
\hline Rank & Gene & Mutation occurrence & Score \\
1 & PIK3CA & 9 & 0.993 \\
2 & CDH1 & 10 & 0.989 \\
3 & IRS2 & 5 & 0.982 \\
4 & IGF1R & 3 & 0.981 \\
5 & PIK3R1 & 2 & 0.947 \\
6 & SYNE1 & 7 & 0.947 \\
7 & HER2 & 3 & 0.906 \\
8 & INSR & 3 & 0.898 \\
9 & KMT2D & 5 & 0.89 \\
10 & PIK3R2 & 3 & 0.887 \\
\hline
\end{tabular}
mutated gene in PILC (11). PYGM was not present in the TumorCare panel and therefore was not identified in our study. However, $P G Y M$ is regulated downstream of IR/IGF1R/IRS2/PI3K signaling, and its mutation provides further evidence for the importance of this pathway in the biology of PILC (15).

IRS2 mutations enhance PILC invasion. The identification of somatic mutations in IRS2 in $29 \%$ of PILC samples is the first report of recurrent IRS2 mutation in any subtype of breast cancer to our knowledge. IRS2 is an adaptor protein for the insulin and IGF-1 receptors, and it mediates their activation of PI3K and MAPK signaling (16). IRS2 mutations are distributed throughout the protein (Figure 3A), as has been observed in other cancer types (17), and this likely reflects the multisite docking function of this adaptor protein. With the exception of the missense V1299I mutation, which is present in the COSMIC database, the remaining IRS2 mutations are novel missense mutations. We assessed the expression of IRS2 in our data set of PILC tumors by IHC staining. All PILC tumors were positive for IRS2 expression, which varied in expression level across the 17 tumors (Figure 3B). In a previous study, we identified three staining patterns for IRS2 in breast cancer. IRS2 was present at the cell membrane, diffusely cytoplasmic, or in a diffuse, cytoplasmic punctate pattern (18). We observed predominantly a punctate cytoplasmic staining pattern for IRS2 in PILC.

A critical issue that arises from our data is whether the IRS2 mutations we identified are function altering. Tumor cell functions that are regulated by IRS2 include glucose uptake, migration, and invasion (16). We initially investigated the effect of IRS2 mutations on invasion because PILC tumors have a high incidence of lymphovascular invasion and lymph node metastasis (1). To investigate if $I R S 2$ mutations enhance invasive potential, WT-IRS2 and individual IRS2 mutants were initially expressed in either SUM-159:IRS1 $1^{-1}, I R S 2^{-1-}$ human breast carcinoma cells (Figure 4A) or PyMT:Irs $1^{-1-}$, Irs $2^{--}$mouse mammary tumor cells (Figure 4G) (19). Both of these cell lines are dependent upon IRS2 for invasion and their lack of IRS2 expression permits analysis of the IRS2 mutations without a background of endogenous expression. Cells were embedded within a Matrigel/collagen I gel that mimics the tumor matrix microenvironment (20). Cells with limited invasion capacity or noninvasive cells grow as spherical colonies, and invasive cells form protrusions into the surrounding matrix. Invasive potential is monitored by the extent and distance of cell branching from the colonies (Figure 4B). Cells expressing vector alone ( $\mathrm{pCDH}$ or $\mathrm{pcDNA}$ ) grew predominantly as spheroids, with minimal invasive branching, and expression of WT-IRS2 increased invasion significantly (Figure 4, C and H). When compared with WT-IRS2, 3 of the IRS2 mutants (S506G, A698T, and S1103L) stimulated a marked and significant increase in the percentage of colonies with extensive invasive branching (Figure 4, $\mathrm{C}$ and $\mathrm{H}$ ) and the distance that these branches invaded into the matrix (Figure 4, D and I). Representative images for each cell line are shown below the graphs.

Contrary to the IRS2-dependent increase in invasion, cell migration was not significantly enhanced by WT-IRS2 expression. Furthermore, no additional significant increases in migration were observed for any of the IRS2 mutants (Figure 4E). Glucose uptake was significantly increased by WT-IRS2 expression; however, the IRS2 mutants did not further enhance uptake above WT-IRS2 levels (Figure 4F). Taken together, these results indicate a selective role for PILC IRS2 mutations in the regulation of invasion.

We next validated the impact of IRS2 mutations on invasion using a pleomorphic lobular carcinoma model. Mice with E-cadherin and p53 inactivation in the mammary epithelium develop invasive, metastatic 
A

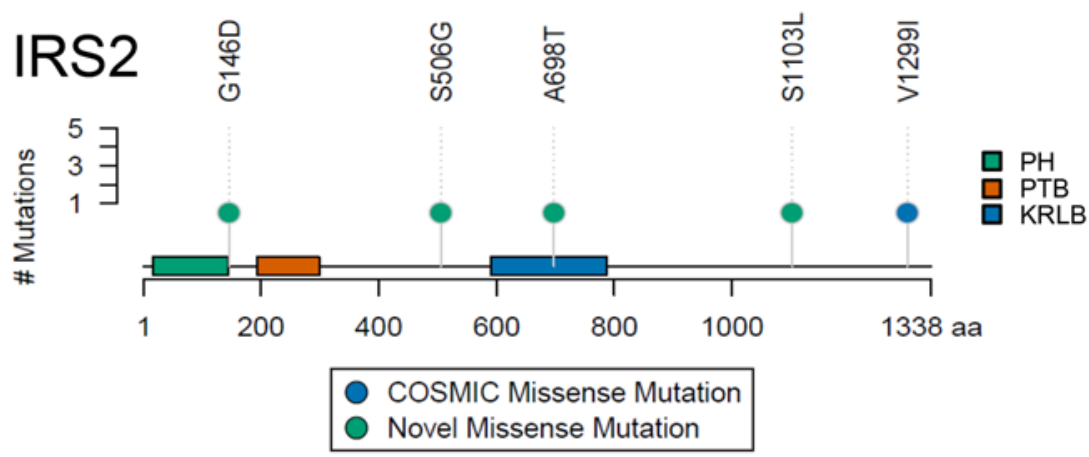

B

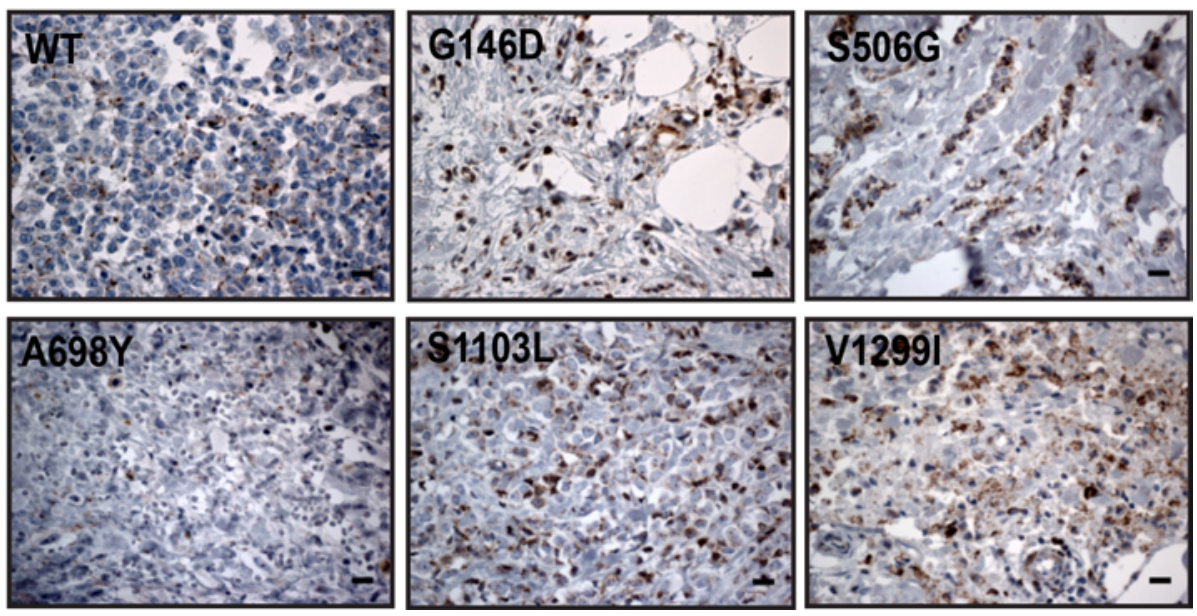

Figure 3. Recurrent IRS2 mutations in pleomorphic invasive lobular carcinoma. (A) Lollipop plot of somatic mutations in IRS2. (B) Representative images of insulin receptor substrate 2 (IRS2) staining in pleomorphic invasive lobular carcinoma tumors. Scale bar: $20 \mu \mathrm{m}$.

tumors that phenocopy human PILC in both primary tumor histology and metastatic dissemination patterns (21). The KEP 1.11 murine PILC cell line derived from these tumors expresses endogenous Irs2 (Figure $5 \mathrm{~A})$, and the invasive potential of these cells is significantly reduced upon shRNA-mediated suppression of Irs2 expression (Figure 5B). This Irs2-mediated invasion is dependent upon upstream receptor activation, as the IR/IGF1R small-molecule inhibitor BMS-754807 blocked invasive branching without effecting colony number (Figure 5, C and D). WT-IRS2 and the IRS2 mutants were expressed in these cells, and the cells were assayed for their ability to invade (Figure $5 \mathrm{E}$ ). A 2-fold increase in the extent of invasive branching was observed upon expression of exogenous WT-IRS2 (Figure 5F). Moreover, the 3 IRS2 mutants (S506G, A698T, and S1103L) that enhanced invasion in SUM-159 and PyMT cells stimulated a significant increase in the percentage of colonies with extensive invasive branching (Figure 5F) and an increase in the distance these branches invaded into the matrix (Figure 5G) when compared with WT-IRS2. Neither migration nor glucose uptake were altered by expression of the IRS2 mutants in the PILC cells (Figure 5, H and I), substantiating the selective role of these mutations in regulating invasion.

\section{Discussion}

We have characterized molecular alterations in PILC that provide insight into the aggressive behavior of this lobular breast cancer variant. Targeted sequencing analysis identified genes that are recurrently mutated in PILC and revealed that PILC is more similar to CILC than to IDC. Specifically, the frequency of molecular alterations in genes that have been reported to discriminate between lobular and ductal carcinoma, including TP53, MYC, GATA3, FOXA1, CDH1, and TBX3, was more similar to the frequency reported for ILC than that for IDC. Recurrent molecular alterations were also identified that distinguish PILC from CILC. Most notably, IRS2, which encodes the IRS2 adaptor protein that mediates signaling downstream 
A

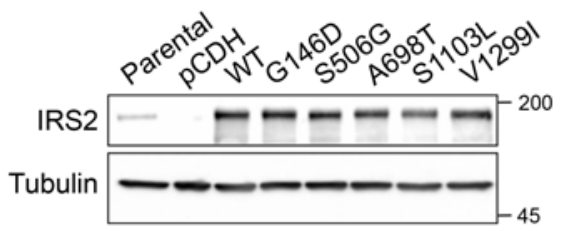

B

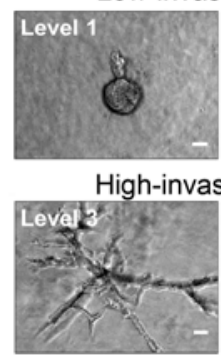

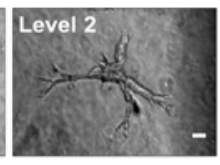

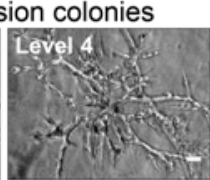

G

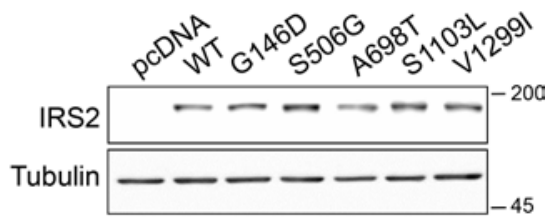

C

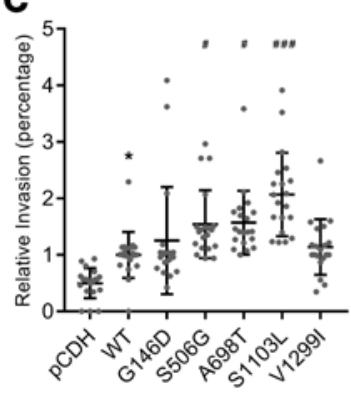

D
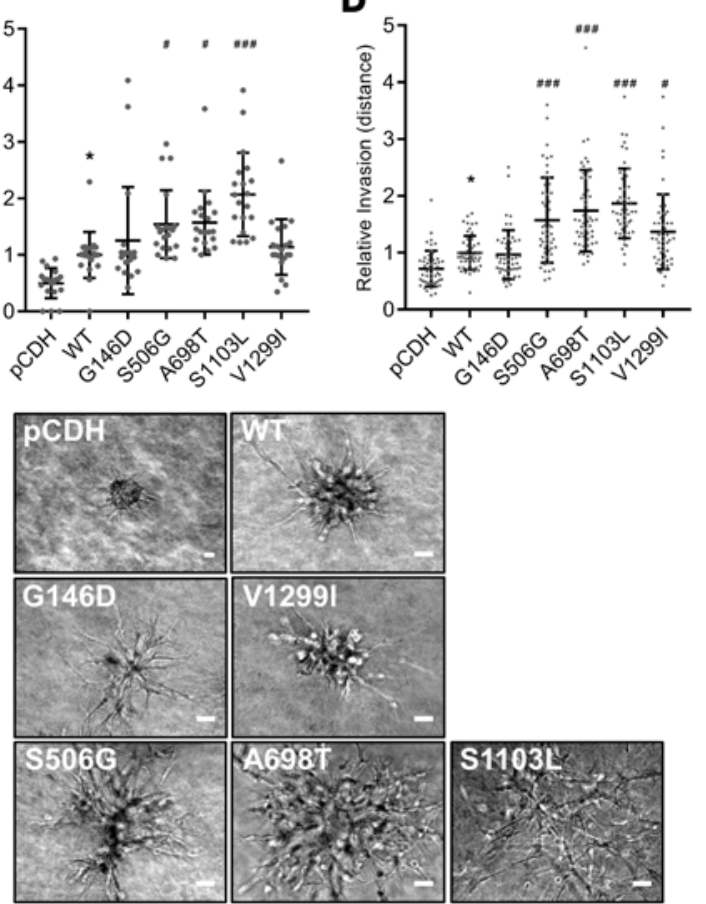

E

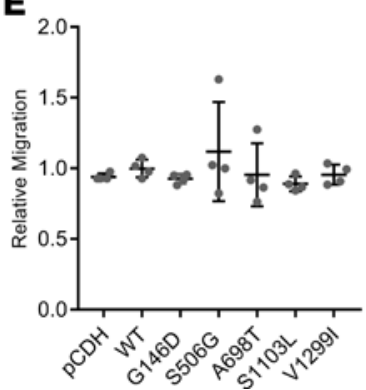

$\mathbf{F}$

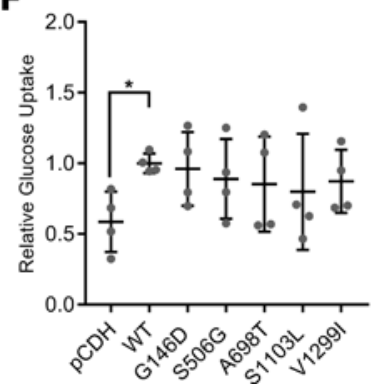

H

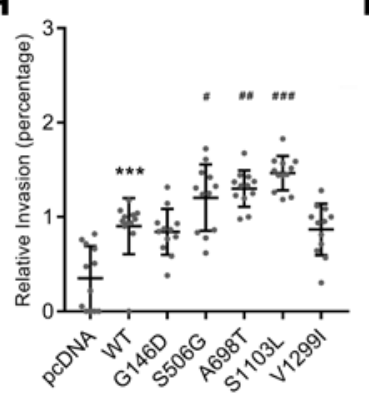

I

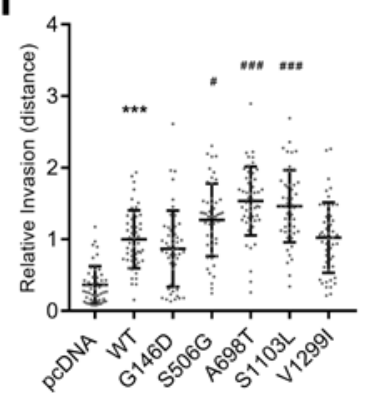

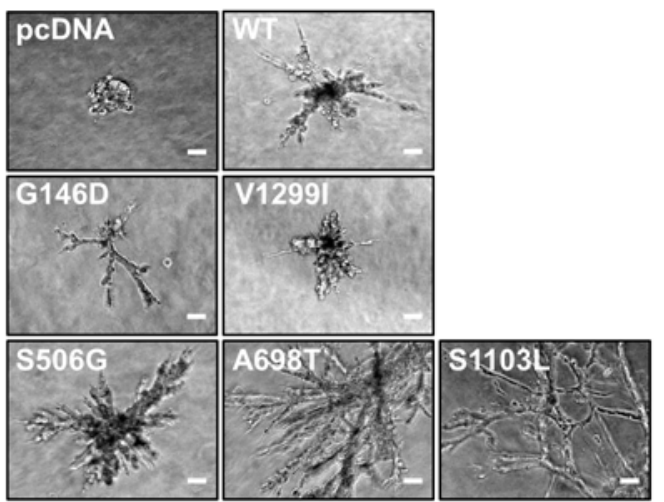

Figure 4. IRS2 mutations linked to invasion. (A) Immunoblots of cell extracts from SUM-159 parental cells and SUM-159:IRS1 expressing empty vector (pCDH), wild-type (WT) human insulin receptor substrate 2 (IRS2), or the IRS2 mutants identified in pleomorphic invasive lobular carcinoma (PILC). (B) Representative images used to score invasive colonies grown in Matrigel/collagen I gels. Cells grown in a Matrigel/ collagen I gel were scored for (C) the extent of invasion (mean \pm SD, $n=20$ wells from 5 independent experiments) or (D) the distance of invasive branching (mean \pm SD, $n=50$ colonies from 1 of 3 representative experiments). Representative images for each cell line are shown. (E) Cell migration assay using Transwell culture chambers (mean \pm SD of 4 independent experiments). (F) Glucose uptake assay (mean \pm SD of 4 independent experiments). (G) Immunoblots of cell extracts from PyMT:Irs1 ${ }^{-1-}$,Irs2 ${ }^{-/-}$cells stably expressing empty vector (pcDNA), wild-type human IRS2 (WT), or the IRS2 mutants identified in PILC. Cells grown in a Matrigel/collagen I gel were scored for (H) the extent of invasion (mean \pm SD, $n=12$ wells from 5 independent experiments) or (I) the distance of invasive branching (mean \pm SD, $n=50$ colonies from 1 of 3 representative experiments). Representative images for each cell line are shown. Student's $t$ test was performed between vector (pCDH or pcDNA) and WT-IRS2, and 1-way ANOVA with Bonferroni post hoc testing was performed between WT-IRS2 and IRS2 mutants. ${ }^{*} P<0.05$, relative to pCDH; ${ }^{* *} P<0.001$, relative to pcDNA; ${ }^{\#} P<0.05,{ }^{\#} P<0.01,{ }^{\# \#} P<0.001$, relative to WT-IRS2. Scale bar: $20 \mu \mathrm{m}$. 
A

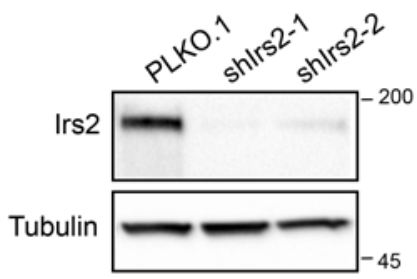

B

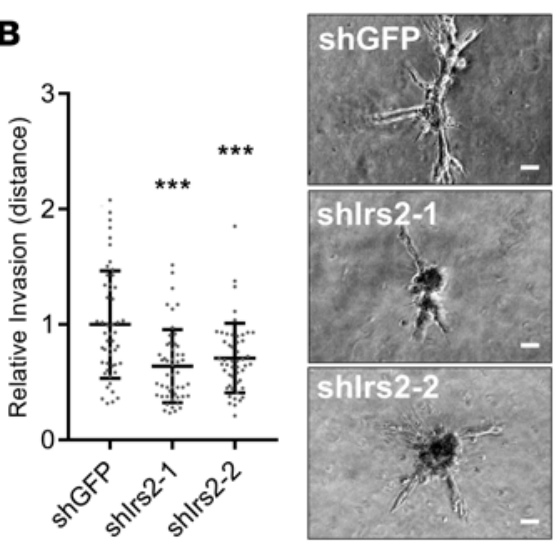

E

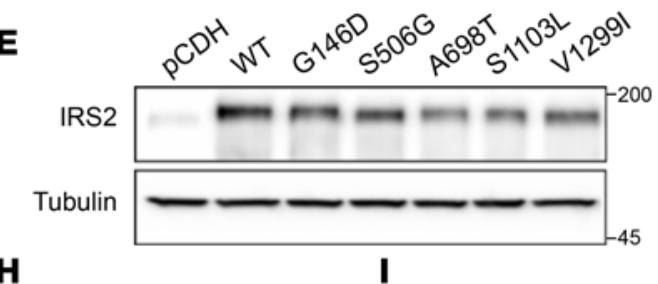

H

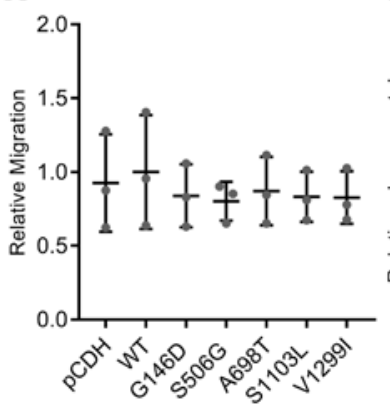

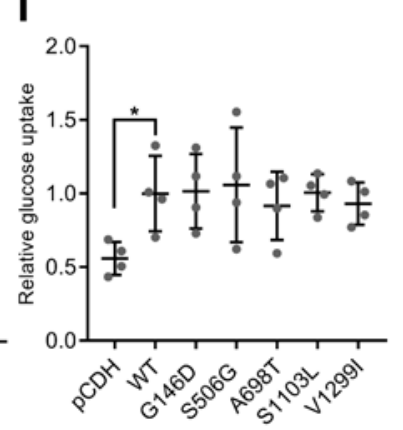

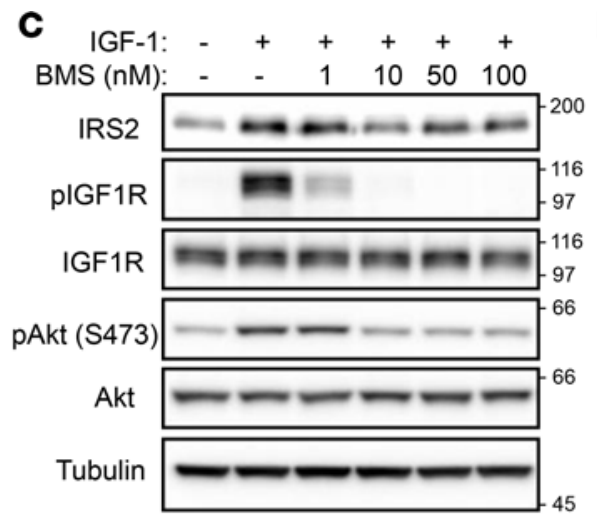

$\mathbf{F}$
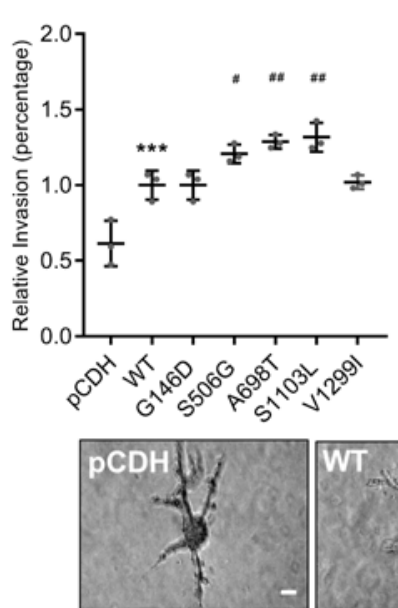

G146D

V1299I
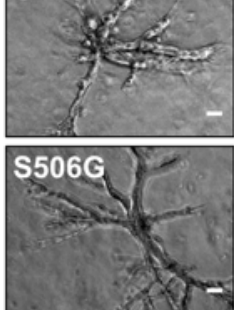

G
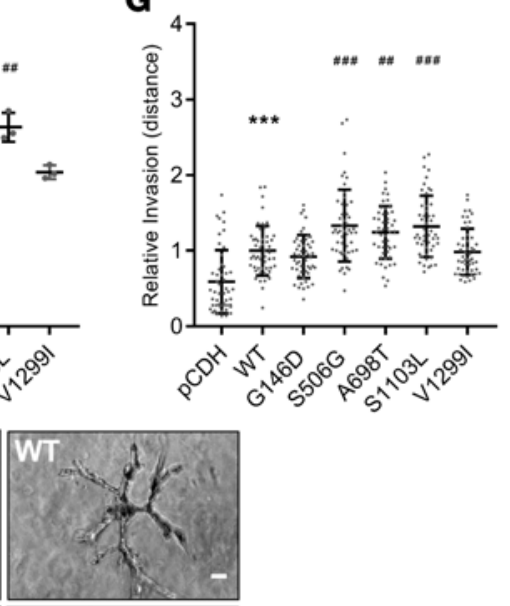

D

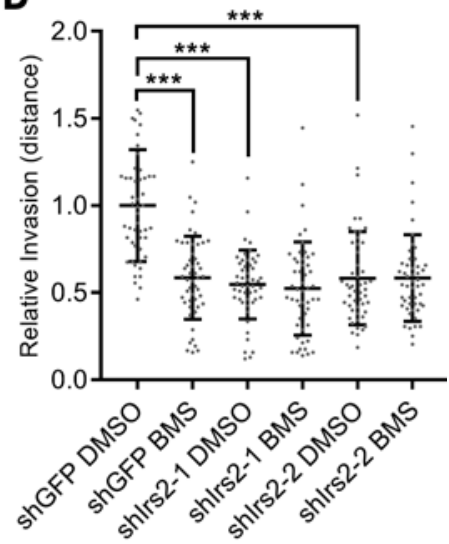

G
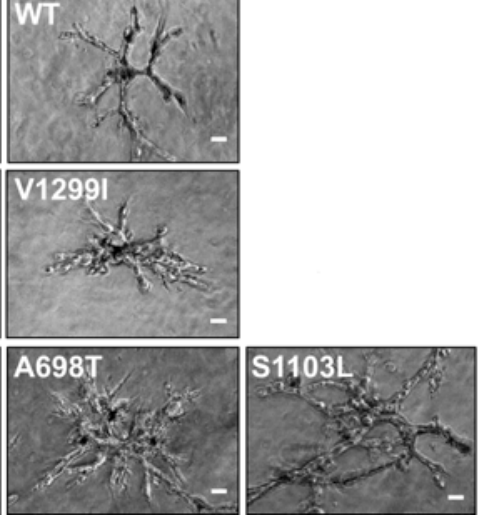

Figure 5. IRS2 mutations linked to invasion in pleomorphic invasive lobular carcinoma cells. (A) Immunoblots of cell extracts from KEP 1.11 murine pleomorphic invasive lobular carcinoma (PILC) cells stably expressing shGFP and 2 independent shRNA targeting Irs2 (shlrs2-1 and shlrs2-2). (B) Cells grown in a Matrigel/collagen I gel were scored for the distance of invasive branching (mean \pm SD, $n=50$ colonies from 1 of 2 representative experiments). (C) KEP 1.11 cells expressing shGFP were serum starved and pretreated with DMSO or BMS-754807 at the concentrations indicated for 4 hours and stimulated with insulin-like growth factor-1 (IGF-1; $50 \mathrm{ng} / \mathrm{ml}$ ) for 30 minutes. Cell extracts containing equivalent amounts of protein were immunoblotted with antibodies specific for insulin receptor substrate 2 (IRS2), pIGF1R (Y1135/1136)/pIR (T1150/1151), IGF1R, pAkt (S473), Akt, or Tubulin. (D) Cells grown in a Matrigel/collagen I gel were treated with DMSO or BMS-754807 (BMS, $100 \mathrm{nM}$ ) on day 3 and scored for the distance of invasive branching on day 7 (mean \pm SD, $n=50$ colonies from a representative experiment). (E) Immunoblots of cell extracts from KEP 1.11 cells stably expressing empty vector (pCDH), wild-type human IRS2 (WT), or the IRS2 mutants identified in PILC. Cells grown in a Matrigel/collagen I gel were scored for (F) the extent of invasion (mean \pm SD of 3 independent experiments) or (G) the distance of invasive branching (mean \pm SD, $n=50$ colonies from 1 of 3 representative experiments). Representative images for each cell line are shown. (H) Cell migration assay using Transwell culture chambers (mean \pm SD of 3 independent experiments). (I) Glucose uptake assay (mean \pm SD of 4 independent experiments). Student's $t$ test was performed between pCDH and WT-IRS2, and 1-way ANOVA with Bonferroni post hoc testing was performed for all other comparisons. ${ }^{*} P<0.05$, ${ }^{* * *} P<0.001$, relative to pLKO.1 or pCDH control; ${ }^{\#}<0.05,{ }^{\# \# P}<0.01,{ }^{\# \# P} P 0.001$, relative to WT-IRS2. Scale bar: $20 \mu \mathrm{m}$.

of the insulin and IGF-1 receptors, is recurrently mutated in PILC. Pathway analysis revealed an important role for the IR/IGFIR/IRS2 signaling pathway in PILC. We validated this analysis by demonstrating that the IRS2 mutations identified in PILC enhance invasion, supporting a contribution of this signaling adaptor to the aggressive behavior of PILC. 
IRS2 mutations have not been detected previously in breast cancer. However, somatic molecular alterations of IRS2 have been reported for other cancer types (22-28). Specifically, amplification of IRS2 was first demonstrated in a study of colorectal cancer (CRC) that investigated molecular alterations in the PI3K signaling pathway, and this amplification was shown in a later study to be mutually exclusive, with both mutations in PIK3CA and IGF2 overexpression $(22,25)$. IRS2 copy number gains were also reported in an analysis of 3,131 cancers across 26 histological cancer types (24) and in small cell lung cancer (28). In more recent genome sequencing studies, IRS2 somatic mutations were identified in CRC as well, although the consequences of these alterations on tumor cell function were not directly investigated (26). Importantly, we identified a selective gain-of-function role for the PILC mutations in the promotion of invasion.

We have previously demonstrated that metastasis is significantly impaired in mouse mammary tumor models in the absence of Irs 2 and enhanced in tumor cells that have increased Irs2 expression and activation $(29,30)$. Our current data reveal that the mutations found in PILC selectively enhance invasion and do not increase migration or glucose uptake. The ability of these mutations to enhance tumor cell invasion highlights a mechanism that could contribute to the high incidence of both regional and distant metastasis associated with PILC. An important question is how do the PILC mutations facilitate the ability of IRS2 to regulate invasion? The IRS adaptor proteins are recruited through $\mathrm{N}$-terminal $\mathrm{PH}$ and PTB domains to activated insulin and IGF-1 receptors where they are phosphorylated by the intrinsic receptor tyrosine kinases to generate SH2-binding sites that mediate recruitment of signaling effectors, in particular PI3K (31). The mutations identified in PILC are spread throughout the protein and do not occur in any of the canonical SH2-binding motifs or the PH and PTB domains. Although the mutations could effect phosphorylation or PI3K recruitment through indirect structural alterations, we did not detect significant differences in the kinetics or level of tyrosine phosphorylation or recruitment of PI3K between WT-IRS2 or any of the IRS2 mutants (data not shown). These results suggest that the mechanism by which invasion is enhanced is likely independent of this canonical IRS signaling pathway. The fact that IRS1 and IRS2 both activate PI3K but only IRS2 promotes invasion, and that 4 of 5 IRS2 mutations overlap with PIK3CA mutations in PILC tumors, also supports an alternative mechanism of action. A reasonable hypothesis to be tested going forward is that the mutations that facilitate invasion enhance the interaction of IRS2 with novel effector proteins. A better understanding of IRS2 structure will be required to model the S506G, A698T, and S1103L mutations to predict how they positively alter IRS2 function to facilitate invasion.

Our identification of genes and pathways that underlie the unique biology of PILC has implications for the clinical management of these highly invasive and metastatic tumors. Our data confirm that HER2 is frequently mutated in PILC and support previous suggestions that PILC tumors that do not have HER2 amplification should undergo sequencing to identify patients who would be responsive to HER2-targeted therapies (32). Previous trials of drugs that target IR or IGF1R signaling have led to disappointing results for many different cancers, including breast cancer, and better biomarkers for selecting patients who would be responsive to these agents are needed $(33,34)$. IRS2 copy number gains were previously shown to enhance the sensitivity of CRC cells to IR/IGF1R inhibition (35). Moreover, high IGF1R activity, as defined by an IGF-1 gene expression signature, correlates with sensitivity of triple-negative breast cancer cells to BMS-754807 (36). Given our finding that targeting the IR/IGF1R pathway inhibits PILC invasion, the mutational status of IRS2 may be exploited to identify PILC patients who are more likely to benefit from drugs that target this pathway to inhibit PILC progression and improve patient outcomes.

\section{Methods}

Targeted exome sequencing and analysis. Patient-matched tumor and normal specimens used in this study were obtained from the University of Massachusetts Medical School Pathology archives. H\&E-stained paraffin tissue sections were reviewed by a board-certified breast pathologist to confirm a diagnosis of PILC. Samples containing $>60 \%$ tumor content were selected for the study, and tissue from 9 adjacent slides was macrodissected for analysis. Normal tissue from each subject was also macrodissected.

DNA extraction and targeted exome sequencing were performed by the Beijing Genomics Institute using their TumorCare panel. Qualified genomic DNA samples were randomly fragmented by Covaris, with a fragment size of between 200 to $300 \mathrm{bp}$. Adapters were then ligated to both ends of the resulting fragments, and purified fragments with insert sizes of approximately $250 \mathrm{bp}$ were selected. The extracted DNA was then amplified by ligation-mediated PCR, purified, and hybridized to Roche NimbleGen SeqCap EZ Exome probes (Roche). The captured libraries of each exome were sequenced on an Illumina Hiseq2000 
system (Illumina). Raw image files were processed by Illumina CASAVA 1.7 software for base calling with default parameters, and the sequences of each library were generated as 100-bp paired-end reads. The quality of the reads was evaluated with fastqc (http://www.bioinformatics.babraham.ac.uk/projects/ fastqc/) and the Genome Analysis Toolkit (GATK) (version 3.5) was used for the variant calling according to GATK best practices (37).

Sequencing reads were aligned to the human reference genome, NCBI build 37 (hg19), using the fast and accurate short-read Burrows-Wheeler alignment algorithm (38). The mapping reads provided $>99.5 \%$ coverage over the targeted exons at an average depth of $824 \times$ coverage (range $382 \times$ coverage to $1,390 \times$ coverage) (Figure 1F). The alignment results were combined into a single BAM format file for each sample, and duplicates were marked using the MarkDuplicates module in the Picard tool (http://broadinstitute. github.io/picard/). Reads marked as duplicates were removed from downstream analysis. Local realignment and quality score recalibration were performed using the GATK with default parameters.

Somatic single nucleotide variants (SNVs) and small insertion/deletions (Indels) were called using the MuTect2 algorithm in GATK3.5 with default parameters. The functional annotation of the called variants was performed using ANNOVAR $(39,40)$. Exome CNVs were statistically processed by exome sequencing-based CNV and loss of heterozygosity detection (ExomeCNV) (41) with the sample admixture rate (normal cell contamination rate) set at 0.3 to reduce the number of false positive calls. After calculating depth-of-coverage and B allele frequencies using the DepthOfCoverage module in GATK3.5, CNVs were called at each exon, which were then combined into segments using circular binary segmentation (42). CNVs with a $\log _{2}$ copy ratio $\geq 1$ or $\leq-1$ were defined as copy number gains (amplification) or losses (deletion), respectively. All candidate SNVs, indels, and CNVs were further manually reviewed using the Integrative Genomics Viewer (43). The confident variant results were then annotated using the Bioconductor package ChIPpeakAnno (version 3.8.2) (44). Genes predicted to have deleterious mutations by Condel (45), damaging mutations by FATHMM (46) or LRT (47), or scaled CADD (48) value $\geq 10$ were included in the filtered data set of somatic mutations (Supplemental Table 2).

The filtered data set of genes containing PILC molecular alterations was analyzed by MUFFINN to identify functionally relevant genes for PILC (13). Results of this analysis are reported as probability scores between 0 and 1. Pathway analysis of the PILC data set was performed using the Reactome database (http://www.reactome.org/) (14).

Cell lines. SUM-159 cells were a gift from Arthur Mercurio (University of Massachusetts Medical School) and were grown in F12 media (Gibco) containing 5\% FBS (MilliporeSigma), $5 \mu \mathrm{g} / \mathrm{ml}$ insulin (MilliporeSigma), and $1 \mu \mathrm{g} / \mathrm{ml}$ hydrocortisone (MilliporeSigma). Mammary tumor cells were isolat-

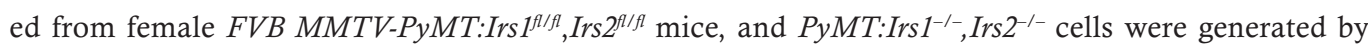
infection with adenoviral Cre recombinase as described previously (19). PyMT mouse mammary tumor cells were grown in low-glucose (1 g/1) DMEM (Corning) containing 10\% FBS. KEP 1.11 murine PILC cells were a gift from Jos Jonkers (Netherlands Cancer Institute, Amsterdam, the Netherlands) and were grown in DMEM/F12 media containing 10\% FBS, Penicillin-Streptomycin (100 U/ml) (Gibco), $5 \mathrm{ng} / \mathrm{ml}$ insulin, $5 \mathrm{ng} / \mathrm{ml}$ epidermal growth factor (MilliporeSigma), and $5 \mathrm{ng} / \mathrm{ml}$ cholera toxin (MilliporeSigma) (21). All cells tested negative for mycoplasma using the Morwell MD Biosciences EZ PCR Mycoplasma Test Kit (catalog 409010).

IRS1/IRS2 double-null SUM-159 cells were generated by CRISPR/Cas9-mediated gene editing. gRNAs were designed using MIT CRISPR DESIGN (http://crispr.mit.edu/) to target an early 5' exon region for either IRS1 (sequence of gRNA: GCATGCTCTTGGGTTTGCGCAGG) or IRS2 (sequence of gRNA: AACCACAGCGTGCGCAAGTGCGG). All gRNAs were subcloned into the pSpCas9(BB)-2A-GFP plasmid (Addgene, 48138) (49). Cells were transfected with the CRISPR plasmid containing the IRS1-gRNA using Lipofectamine 2000 (Invitrogen) and sorted by flow cytometry for the GFPhi pop-

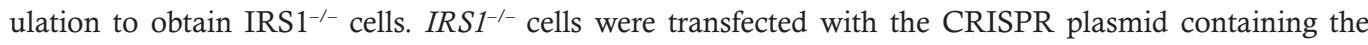
IRS2-specific gRNA and sorted for GFP high cells to generate SUM-159:IRS1 ${ }^{-1-}, I R S 2^{-1-}$ cells. Wild-type IRS2 and the IRS2 mutants were subcloned into the pCDH-CMV-MCS-EF1-puro lentiviral vector (System

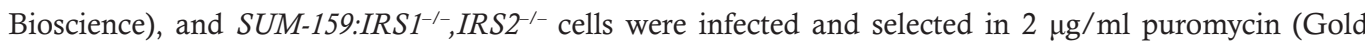

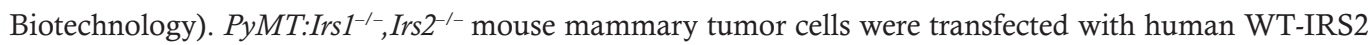
and the IRS2 mutants in pcDNA3.1 and selected in $500 \mu \mathrm{g} / \mathrm{ml} \mathrm{G418} \mathrm{(Gibco).} \mathrm{Lentiviral} \mathrm{vectors} \mathrm{(pLKO.1)}$ containing small hairpin RNAs (shRNAs) targeting the open reading frame region of Irs2 were obtained from Open Biosystems (TRCN0000055108 and TRCN0000055110). 
For IGF1R/IR inhibition, cells were serum starved and pretreated with BMS-754807 for 4 hours before stimulation for 30 minutes with human recombinant IGF-1 (50 ng/ml) (R\&D Systems). Cells were solubilized at $4^{\circ} \mathrm{C}$ in a $20 \mathrm{mM}$ Tris, $\mathrm{pH} 7.4$ buffer containing $1 \%$ nonidet $\mathrm{P}-40,150 \mathrm{mM}$ sodium chloride, $10 \%$ glycerol, $10 \mathrm{mM}$ sodium fluoride, $1 \mathrm{mM}$ sodium orthovanadate, and protease inhibitors (Roche). The dual IGF1R/IR inhibitor BMS-754807 was obtained from Selleckchem (catalog S1124).

Immunoblotting. Cell extracts containing equivalent amounts of total protein were resolved by SDSPAGE and transferred to nitrocellulose membranes. The membranes were blocked for 1 hour with a $50 \mathrm{mM}$ Tris buffer, $\mathrm{pH} 7.5$, containing $0.15 \mathrm{M} \mathrm{NaCl}, 0.05 \%$ Tween 20, and 5\% (wt/vol) dry milk and incubated overnight at $4^{\circ} \mathrm{C}$ in the same blocking buffer containing primary antibodies. After washing, the membranes were incubated for 1 hour in blocking buffer containing peroxidase-conjugated secondary antibodies. Proteins were detected by enhanced chemiluminescence (Bio-Rad). Antibodies used for immunoblotting include IRS2 (Cell Signaling, 4502), pIGF1R(Y1135/1136)/pIR(T1150/1151) (Cell Signaling, 3024), IGF1R (Cell Signaling, 3027), pAkt (S473) (Cell Signaling, 9271), Akt (Cell Signaling, 9272), Tubulin (MilliporeSigma, T5168), and peroxidase-conjugated goat anti-rabbit IgG (Jackson ImmunoResearch Laboratories Inc., 111-035-144) or goat anti-mouse IgG (Jackson ImmunoResearch Laboratories Inc., 711-035-151).

Immunohistochemistry. Formalin-fixed, paraffin-embedded tissue sections ( $5 \mu \mathrm{M})$ were deparaffinized and rehydrated, and antigen retrieval was carried out in $10 \mathrm{mM}$ sodium citrate, $\mathrm{pH} 6.0$, with heating in a steamer for 60 minutes. Tissue sections were blocked with a Avidin/Biotin Blocking Kit (Vector Laboratories) followed by $1 \times$ Casein Solution (Vector Laboratories, SP-5020) for 1 hour. Sections were stained with rabbit monoclonal IRS2 (1:700; Abcam, EP976Y) and counterstained with hematoxylin. Stained tumor sections were viewed on an Olympus BX41 light microscope (Olympus). Photomicrographs were obtained using an Evolution MPColor camera (Media Cybernetics).

Invasion assay. 2,500 cells were suspended in a mixture of Matrigel ( $2 \mathrm{mg} / \mathrm{ml})$ and collagen I ( $1 \mathrm{mg} /$ $\mathrm{ml}$ ) and plated over a base gel layer of the same matrix composition in 8 -well chamber glass slides. The gels were overlaid with complete serum-containing medium, which was changed every 2 days for 7-8 days. DMSO or BMS-754807 (100 nM) was added to the wells on day 3. Colonies were imaged and scored for the extent of cell invasion/branching using a Nikon Diaphot 300 microscope. Cell invasion was categorized into 4 levels, none (level 1), low (level 2), medium (level 3), and high (level 4), depending upon the extent of tumor cell dissemination from the colonies into the surrounding matrix (Figure 4B). Invasion was scored as the percentage of highly invasive colonies (level 3 and 4) in each well. The distance of invasion was determined by measuring from the center of the colonies to the outer tip of the invasive branches. Relative invasion was measured as the fold change in invasion (percentage or distance) compared with cells expressing WT-IRS2. Matrigel and collagen I were obtained from Corning Discovery Labware Inc. (354230 and 354236, respectively).

Migration assay. Migration assays were performed using Transwell chambers (Corning) as described previously (50). Briefly, cells $\left(1 \times 10^{5}\right)$ were resuspended in F12 medium containing $0.5 \%$ FBS and seeded in triplicate in the upper wells of the Transwell chambers. F12 medium containing $0.5 \%$ FBS and $40 \mu \mathrm{g} / \mathrm{ml}$ collagen I was added to the lower wells. After 2.5 hours, cells that migrated to the lower surface of the membrane were fixed with ice-cold 100\% methanol, and the fixed membranes were mounted on glass slides using Vectashield mounting medium with DAPI (Vector Laboratories). Migration was quantified by counting the number of stained nuclei and normalized to that of vector control cells to calculate relative migration.

Glucose uptake assay. Cells were grown in 12-well plates to near confluence, washed with PBS, and then incubated in $0.1 \%$ BSA/DMEM ( $1 \mathrm{~g} / 1$ glucose) for 24 hours. Glucose levels in the media were measured using a glucose (GO) Assay kit (MilliporeSigma, GAGO20) according to the manufacturer's instructions. Glucose uptake was expressed as a rate measurement $(\mathrm{mM} / \mathrm{mg} / \mathrm{h})$ normalized to total protein in each well.

Statistics. Statistical analysis between vector control (pCDH or pcDNA) and IRS2-WT was performed using a 2-tailed unpaired Student's $t$ test. Statistical analysis between IRS2-WT and the IRS2 mutants was performed using 1-way ANOVA followed by Bonferroni's multiple comparisons testing. All statistical analysis was performed using Prism7, Graphpad. A 2-sided $P$ value of less than 0.05 was considered to indicate statistical significance.

Study approval. The study was approved and performed in accordance with standards established by the University of Massachusetts Medical School Institutional Review Board, consistent with applicable national and state laws and regulations. Tissue was acquired through a waiver of consent for retrospective 
data collection. Primary sequencing data have been deposited in the European Genome-phenome Archive under controlled access (accession EGAD00001003995).

\section{Author contributions}

BMW and LMS were involved in the conception and design of the project and wrote the manuscript. SZ, ANMO, and JJ were involved with the development of methodology and the acquisition and analysis of data. DK, KT, and LH were involved in the acquisition of samples and the analysis of data. JY, CCH, and LJZ were involved in the analysis of data. All authors reviewed and approved the final version of the manuscript.

\section{Acknowledgments}

We thank Rebecca Stolarczyk for assistance with data analysis and Art Mercurio for helpful comments on the manuscript. This work was supported by NIH grant CA142782 (to LMS), Avon Foundation grant 02-2016-025 (to LMS and BMW), and the University of Massachusetts Medical School Faculty Diversity Scholar Program (to BMW). The content is solely the responsibility of the authors and does not necessarily represent the official views of the NIH.

Address correspondence to: Leslie M. Shaw, Department of Molecular, Cell and Cancer Biology, University of Massachusetts Medical School, 364 Plantation Street, Worcester, Massachusetts 01605, USA. Phone: 508.856.8675; Email: leslie.shaw@umassmed.edu.

BMW's present address is: Department of Surgery, Albany Medical College, Albany, New York, USA.

1. Al-Baimani K, Bazzarelli A, Clemons M, Robertson SJ, Addison C, Arnaout A. Invasive pleomorphic lobular carcinoma of the breast: pathologic, clinical, and therapeutic considerations. Clin Breast Cancer. 2015;15(6):421-425.

2. Dabbs DJ, et al. Lobular neoplasia of the breast revisited with emphasis on the role of E-cadherin immunohistochemistry. Am $J$ Surg Pathol. 2013;37(7):e1-11.

3. Simpson PT, et al. Molecular profiling pleomorphic lobular carcinomas of the breast: evidence for a common molecular genetic pathway with classic lobular carcinomas. J Pathol. 2008;215(3):231-244.

4. Sikora MJ, Jankowitz RC, Dabbs DJ, Oesterreich S. Invasive lobular carcinoma of the breast: patient response to systemic endocrine therapy and hormone response in model systems. Steroids. 2013;78(6):568-575.

5. Jung SP, et al. Invasive pleomorphic lobular carcinoma of the breast: clinicopathologic characteristics and prognosis compared with invasive ductal carcinoma. J Breast Cancer. 2012;15(3):313-319.

6. Bamford S, et al. The COSMIC (Catalogue of Somatic Mutations in Cancer) database and website. Br J Cancer. 2004;91(2):355-358

7. Ciriello G, et al. Comprehensive molecular portraits of invasive lobular breast cancer. Cell. 2015;163(2):506-519.

8. Desmedt C, et al. Genomic characterization of primary invasive lobular breast cancer. J Clin Oncol. 2016;34(16):1872-1881.

9. Michaut M, et al. Integration of genomic, transcriptomic and proteomic data identifies two biologically distinct subtypes of invasive lobular breast cancer. Sci Rep. 2016;6:18517.

10. Engelman JA, Luo J, Cantley LC. The evolution of phosphatidylinositol 3-kinases as regulators of growth and metabolism. Nat Rev Genet. 2006;7(8):606-619.

11. Dieci MV, et al. Whole exome sequencing of rare aggressive breast cancer histologies. Breast Cancer Res Treat. 2016;156(1):21-32.

12. Boelens MC, et al. PTEN loss in e-cadherin-deficient mouse mammary epithelial cells rescues apoptosis and results in development of classical invasive lobular carcinoma. Cell Rep. 2016;16(8):2087-2101.

13. Cho A, Shim JE, Kim E, Supek F, Lehner B, Lee I. MUFFINN: cancer gene discovery via network analysis of somatic mutation data. Genome Biol. 2016;17(1):129.

14. Joshi-Tope G, et al. Reactome: a knowledgebase of biological pathways. Nucleic Acids Res. 2005;33(Database issue):D428-D432.

15. Syed NA, Khandelwal RL. Reciprocal regulation of glycogen phosphorylase and glycogen synthase by insulin involving phosphatidylinositol-3 kinase and protein phosphatase-1 in HepG2 cells. Mol Cell Biochem. 2000;211(1-2):123-136.

16. Mardilovich K, Pankratz SL, Shaw LM. Expression and function of the insulin receptor substrate proteins in cancer. Cell Commun Signal. 2009;7:14

17. Cerami E, et al. The cBio cancer genomics portal: an open platform for exploring multidimensional cancer genomics data. Cancer Discov. 2012;2(5):401-404.

18. Clark JL, et al. Membrane localization of insulin receptor substrate-2 (IRS-2) is associated with decreased overall survival in breast cancer. Breast Cancer Res Treat. 2011;130(3):759-772.

19. Landis J, Shaw LM. Insulin receptor substrate 2-mediated phosphatidylinositol 3-kinase signaling selectively inhibits glycogen synthase kinase $3 \beta$ to regulate aerobic glycolysis. J Biol Chem. 2014;289(26):18603-18613.

20. Nguyen-Ngoc KV, Shamir ER, Huebner RJ, Beck JN, Cheung KJ, Ewald AJ. 3D culture assays of murine mammary branching morphogenesis and epithelial invasion. Methods Mol Biol. 2015;1189:135-162.

21. Derksen PW, et al. Mammary-specific inactivation of E-cadherin and p53 impairs functional gland development and leads to pleomorphic invasive lobular carcinoma in mice. Dis Model Mech. 2011;4(3):347-358.

22. Parsons DW, et al. Colorectal cancer: mutations in a signalling pathway. Nature. 2005;436(7052):792. 
23. Leary RJ, et al. Integrated analysis of homozygous deletions, focal amplifications, and sequence alterations in breast and colorectal cancers. Proc Natl Acad Sci USA. 2008;105(42):16224-16229.

24. Beroukhim R, et al. The landscape of somatic copy-number alteration across human cancers. Nature. 2010;463(7283):899-905

25. Cancer Genome Atlas Network. Comprehensive molecular portraits of human breast tumours. Nature. 2012;490(7418):61-70

26. Bertotti A, et al. The genomic landscape of response to EGFR blockade in colorectal cancer. Nature. 2015;526(7572):263-267

27. Day E, et al. IRS2 is a candidate driver oncogene on 13q34 in colorectal cancer. Int J Exp Pathol. 2013;94(3):203-211.

28. George J, et al. Comprehensive genomic profiles of small cell lung cancer. Nature. 2015;524(7563):47-53.

29. Nagle JA, Ma Z, Byrne MA, White MF, Shaw LM. Involvement of insulin receptor substrate 2 in mammary tumor metastasis. Mol Cell Biol. 2004;24(22):9726-9735.

30. Ma Z, Gibson SL, Byrne MA, Zhang J, White MF, Shaw LM. Suppression of insulin receptor substrate 1 (IRS-1) promotes mammary tumor metastasis. Mol Cell Biol. 2006;26(24):9338-9351.

31. White MF. IRS proteins and the common path to diabetes. Am J Physiol Endocrinol Metab. 2002;283(3):E413-E422.

32. Lien HC, Chen YL, Juang YL, Jeng YM. Frequent alterations of HER2 through mutation, amplification, or overexpression in pleomorphic lobular carcinoma of the breast. Breast Cancer Res Treat. 2015;150(2):447-455.

33. King H, Aleksic T, Haluska P, Macaulay VM. Can we unlock the potential of IGF-1R inhibition in cancer therapy? Cancer Treat Rev. 2014;40(9):1096-1105.

34. Iams WT, Lovly CM. Molecular Pathways: Clinical applications and future direction of insulin-like growth factor-1 receptor pathway blockade. Clin Cancer Res. 2015;21(19):4270-4277.

35. Huang F, et al. IRS2 copy number gain, KRAS and BRAF mutation status as predictive biomarkers for response to the IGF-1R/ IR inhibitor BMS-754807 in colorectal cancer cell lines. Mol Cancer Ther. 2015;14(2):620-630.

36. Litzenburger BC, et al. High IGF-IR activity in triple-negative breast cancer cell lines and tumorgrafts correlates with sensitivity to anti-IGF-IR therapy. Clin Cancer Res. 2011;17(8):2314-2327.

37. DePristo MA, et al. A framework for variation discovery and genotyping using next-generation DNA sequencing data. Nat Genet. 2011;43(5):491-498.

38. Li H, Durbin R. Fast and accurate short read alignment with Burrows-Wheeler transform. Bioinformatics. 2009;25(14):1754-1760.

39. Wang K, Li M, Hakonarson H. ANNOVAR: functional annotation of genetic variants from high-throughput sequencing data. Nucleic Acids Res. 2010;38(16):e164.

40. Yang H, Wang K. Genomic variant annotation and prioritization with ANNOVAR and wANNOVAR. Nat Protoc. 2015;10(10):1556-1566.

41. Sathirapongsasuti JF, et al. Exome sequencing-based copy-number variation and loss of heterozygosity detection: ExomeCNV. Bioinformatics. 2011;27(19):2648-2654.

42. Olshen AB, Venkatraman ES, Lucito R, Wigler M. Circular binary segmentation for the analysis of array-based DNA copy number data. Biostatistics. 2004;5(4):557-572.

43. Robinson JT, et al. Integrative genomics viewer. Nat Biotechnol. 2011;29(1):24-26.

44. Zhu LJ, et al. ChIPpeakAnno: a Bioconductor package to annotate ChIP-seq and ChIP-chip data. BMC Bioinformatics. 2010;11:237.

45. González-Pérez A, López-Bigas N. Improving the assessment of the outcome of nonsynonymous SNVs with a consensus deleteriousness score, Condel. Am J Hum Genet. 2011;88(4):440-449.

46. Shihab HA, et al. Predicting the functional, molecular, and phenotypic consequences of amino acid substitutions using hidden Markov models. Hum Mutat. 2013;34(1):57-65.

47. Chun S, Fay JC. Identification of deleterious mutations within three human genomes. Genome Res. 2009;19(9):1553-1561.

48. Kircher M, Witten DM, Jain P, O'Roak BJ, Cooper GM, Shendure J. A general framework for estimating the relative pathogenicity of human genetic variants. Nat Genet. 2014;46(3):310-315.

49. Ran FA, Hsu PD, Wright J, Agarwala V, Scott DA, Zhang F. Genome engineering using the CRISPR-Cas9 system. Nat Protoc. 2013;8(11):2281-2308.

50. Rohatgi RA, et al. Beclin 1 regulates growth factor receptor signaling in breast cancer. Oncogene. 2015;34(42):5352-5362.

51. Shannon P, et al. Cytoscape: a software environment for integrated models of biomolecular interaction networks. Genome Res. 2003;13(11):2498-2504. 